\title{
The relationship between postmenopausal women's sclerostin levels and their bone density, age, body mass index, hormonal status, and smoking and consumption of coffee and dairy products
}

\author{
Muberra Namlı Kalem ${ }^{1}$
}

Received: 3 May 2017/Accepted: 8 May 2017/Published online: 16 May 2017

(C) Springer-Verlag Berlin Heidelberg 2017

Dear Editor and Dr. Tomoyuki Kawada,

We are grateful for your interest in and constructive recommendations for our study. We made additions to our statistical study in accordance with your recommendations and are presenting the new statistically analysis results below.

Single-parameter analyses and linear regression analysis were conducted, respectively, with the sclerostin, which is the only dependent parameter, for all parameters in the study, and the parameters to be included in the model were specified. These parameters were found to be coffee, cigarette, milk and dairy products, BMI, and menopause year. As there was a significant difference between the categories of the menopause year for the BMI parameter, these two parameters were included in the linear regression analysis individually along with other parameters.

In the model in which menopause year, coffee, cigarette, and milk and dairy products were included as independent parameters and sclerostin as the dependent parameter, only the menopause year ( $p=0.018$ ) was found to be significant. The model of the regression was found to be sclerostin $=80.523-12.761 \times$ menopause year. The $R^{2}$ value for this model was found to be 0.070 , and it can be accordingly concluded that the parameter of menopause year alone explains only $7 \%$ of the change in sclerostin.

In the model in which BMI, coffee, cigarette, and milk and dairy products were included as independent parameters and sclerostin as the dependent parameter, only BMI ( $p=0.001)$ was found to be significant. The model of the regression was found to be sclerostin $=-200.600+10.150 \times$ BMI. The $R^{2}$ value for this model was found to be 0.368 , and it can be accordingly concluded that the parameter of BMI alone explains only $36.8 \%$ of the change in sclerostin.

In conclusion, we believe how the correlation we put forth between BMI and sclerostin is supported with the multiple linear regression analysis added value to the study. We, again, would like to thank for your contribution.

Kind regards,

Müberra Namlı Kalem

03 May 2017

Turkey

Compliance with ethical standards

Conflict of interest The authors declare that they have no conflict of interest.
Muberra Namlı Kalem

muberranamli@hotmail.com

1 Department of Obstetrics and Gynecology, Liv Hospital Ankara, Ankara, Turkey 